\title{
Additive Protective Effects of Luteolin and Pyruvate against 6-Hydroxydopamine and 3-Hydroxykynurenine Induced Neurotoxicity in SH-SY5Y Cells*
}

\author{
Natalia Wszelaki, Matthias F. Melzig \\ Institut für Pharmazie/Pharmazeutische Biologie, Freie Universität Berlin, Berlin, Germany. \\ Email: melzig@zedat.fu-berlin.de \\ Received April 16 $6^{\text {th }}, 2013$; revised May 28 $8^{\text {th }}, 2013$; accepted June $10^{\text {th }}, 2013$ \\ Copyright (C) 2013 Natalia Wszelaki, Matthias F. Melzig. This is an open access article distributed under the Creative Commons Attribu- \\ tion License, which permits unrestricted use, distribution, and reproduction in any medium, provided the original work is properly cited.
}

\begin{abstract}
Oxidative stress has been implicated as one of the causes in cell death in many neurodegenerative disorders. Due to antioxidative properties in vitro, the use of flavonoids and other polyphenolic compounds synthesised by plants are considered to be a promising strategy to prevent Alzheimer's disease and Parkinsons's disease. In the present study, we tested protective effects of some polyphenols and sodium pyruvate on 6-hydroxydopamine (6-OHDA), salsolinol and 3-hydroxykynurenine (3-HK) induced neurotoxicity in human neuroblastoma SH-SY5Y cells. We found that luteolin prevented from 6-OHDA and 3-HK induced cell viability reduction and that one of the mechanisms involved in the neuroprotective process was the ability to increase the level of cellular ATP. However, luteolin was ineffective against salsolinol-induced toxicity. Neither pre-treatment with flavonoids nor simultaneous addition had any protective effects on 6-OHDA, salsolinol or 3-HK induced neurotoxicity. Interestingly, both pre-treatment and co-treatment with pyruvate provided protection against 6-OHDA, salsolinol or 3-HK induced toxicity. Moreover, luteolin and sodium pyruvate, administered together, acted additively, so to achieve the same effect, lower concentrations were needed. The ability of luteolin and sodium pyruvate to reduce toxicity of 6-OHDA and 3-HK in SH-SY5Y cells may be related to two different neuroprotective mechanisms and the capability to penetrate into the cell.
\end{abstract}

Keywords: Flavonoids; Oxidative Stress; Pyruvate; ATP; Luteolin; Neurotoxicity

\section{Introduction}

Oxidative stress is induced by an imbalance between the production of reactive oxygen species (ROS) and the detoxification of reactive intermediates in mitochondria and has been strongly implicated as one of the causes and triggering factors of cell death. Accumulation of ROS and decreased antioxidant capability can lead to damage of cell lipids, proteins, nucleic acids and might disturb mitochondrial functions, which results in initiating apoptotic and necrotic cell death. There is extensive literature on mitochondrial dysfunction and its association with different neurodegenerative diseases such as Alzheimer's disease (AD), Parkinson's disease (PD) or Huntington's disease $[1,2]$. ROS are highly reactive and toxic reduced oxygen forms such as hydrogen peroxide $\left(\mathrm{H}_{2} \mathrm{O}_{2}\right)$, superoxide radical $\left(\mathrm{O}_{2}^{-}\right)$and hydroxyl radical $\left(\mathrm{OH}^{-}\right)$continuously generate in the mitochondrial respiratory chain,

\footnotetext{
*Authors declare no conflicts of interest.
}

in which energy is produced in the form of adenosine-5'-triphosphate (ATP). Moreover, neurons are especially vulnerable to elevated ROS or decreased ATP level, due to the fact that those cells are rich in mitochondria and have a high demand for ATP. Intracellular ROS generation can be induced by a number of diverse insults, e.g. salsolinol, 6-OHDA, 3-HK, A $\beta$, 1-methyl-4phenyl-1,2,3,6-tetrahydropyridine-(MPTP) or rotenone [37]. Physiologically the only available defence against ROS in mitochondria are enzymes (for example superoxide dismutase, catalase, peroxidase) or low molecular weight oxidants (e.g. chelating agents, glutathione, NADPH). Externally generated oxidative stress may be reduced through agents from dietary sources. However, in order to reach equilibrium between ROS production and detoxification in brain cells the antioxidant strategies have been proposed. Since flavonoids, polyhydroxylated phytochemicals and common dietary components present in fruits, vegetables and beverages like tea or wine, have 
been proven to posses strong anti-oxidative, anti-inflammatory and other biological properties in vitro [8], the use of flavonoids and other polyphenolic compounds synthesised by plants has been considered to be a promising strategy to protect from neurodegenerative diseases including $\mathrm{AD}$ and $\mathrm{PD}$.

On the other hand, it has been noted that several antioxidants, such as catalase, vitamin E, ascorbic acid, pyruvate, glutathione, $\mathrm{N}$-acetyl cysteine, protected from 6-OHDA-induced toxicity in cells [4,9-11] and that their bioavailability were reported higher than flavonoids.

Sodium pyruvate, an endogenous low molecular weight antioxidant and energy substrate was found to protect neuronal cells through direct acting in mitochondria by decreasing generation of ROS and stabilization of mitochondrial membrane potential [12]. In various experimental cell models it was also found to protect against such insults as $\beta$-amyloid and 6-OHDA $[10,13]$.

In the present study, we tested potential protective effects of apigenin, amentoflavone, chrysine, (-) epigallocatechin-3-gallate, isorhamnetin, kaempferol, luteolin, luteolin-7-glucoside, myricetin, naringenin, quercetin, resveratrol, rosmarinic acid, rutin, saponarin, sinensetin and sodium pyruvate in human neuroblastoma cells SHSY5Y exposed to different neurotoxic agents such as salsolinol, 6-OHDA and 3-HK.

\section{Materials and Methods}

\subsection{Reagents and Tested Substances}

Dulbecco's Modified Eagle's Medium (DMEM), fetal bovine serum (FBS), phosphate buffered saline (PBS), Trypsin-EDTA, sodium pyruvate were purchased from Biochrom, Berlin, Germany. Amentoflavone, apigenin, isorhamnetin, kaempferol, luteolin, luteolin-7-glucosid, naringenin, sinensetin, rosmarinic acid and were obtained from Roth, Karlsruhe, Germany; chrysine, epigallocatechin-3-gallate (EGCG), myricetin, quercetin, resveratrol, 3-hydroxykynurenine, 6-hydroxydopamine, $( \pm)$ salsolinol, 3-(4,5-dimethylthiazol-2-yl)-2,5-diphenyltetrazolium bromide (MTT) were from Sigma, St. Louis, MO. Saponarin was a generous gift from Maya Yotova (Medical University of Sofia, Bulgaria).

Flavonoids and rosmarinic acid were solubilised in dimethyl sulfoxide (DMSO), stored in the dark at $-20^{\circ} \mathrm{C}$, and diluted in medium before use. The final concentration of DMSO was limited to $0.5 \%$ and did not affect cell viability.

\subsection{Cell Cultures}

Human neuroblastoma cell line SH-SY5Y was obtained from Deutsche Sammlung von Mikroorganismen und Zellkulturen (DMSZ), Braunschweig, Germany. SH-SY5Y cell cultures were grown in DMEM supplemented with $15 \%$ FBS. All cells were maintained at $37^{\circ} \mathrm{C}$ in a humidified $5 \% \mathrm{CO}_{2} /$ air atmosphere. The cells were transferred to low serum media (1\% FBS) 2 hours prior to the treatment. Cells were weekly passaged (Trypsin-EDTA, Biochrom, Germany). Cell number was measured using CASY cell counter (Schärfe System, Reutlingen, Germany). Tests were carried out in 96-well microplates, and the density of $5 \times 10^{4}$ (SH-SY5Y) cells per well was considered in the screening experiment. Cells were incubated $24 \mathrm{~h}$ before the drug was added, and the drug remained in contact with the cells for $24 \mathrm{~h}$ or $48 \mathrm{~h}$.

\subsection{Cell Viability Assay/(MTT) Reduction Assay}

For the viability assay, cells were plated at a density of 5 $\times 10^{4} \mathrm{SH}-\mathrm{SY} 5 \mathrm{Y}$ cells per well on $96-$-well plates in 100 $\mu 1$ of DMEM containing $1 \%$ FBS. One day after plating, amentoflavone, apigenin, chrysine, epigallocatechin 3gallate, isorhamnetin, kaempferol, luteolin, luteolin-7glucosid, saponarin, sinensetin, naringenin, myricetin, quercetin, rosmarinic acid, rutin and sodium pyruvate were added at various concentrations to the cells for 24 hours. 3-HK $(500 \mu \mathrm{M})$, 6-OHDA $(75 \mu \mathrm{M})$ or salsolinol $(500 \mu \mathrm{M})$ were given to the medium simultaneously or after 24 hours of pre-treatment with tested compounds for $24 \mathrm{~h}$. As control, cells were incubated with $0.5 \%$ of DMSO, which did not influence cell viability, assessed using the modified MTT assay [14]. Briefly, MTT was added to the cells after treatment in 96-well plates. Cells were incubated for $2 \mathrm{~h}$ with MTT solution $(5 \mathrm{mg} / \mathrm{ml})$. The formazan formed was dissolved in DMSO and the absorbance was measured by a multiplate reader at 570 $\mathrm{nm}$ wavelength and at $620 \mathrm{~nm}$ as reference wavelength (Tecan, Austria). All experiments were done in triplicate. Results were expressed as percentage of control.

\subsection{Measurement of Cellular ATP Content}

Cellular ATP concentrations were assessed by the CellTiter-Glo cell viability assay, according to the manufacturer's instructions. Briefly, the assay is based on measurement of luminescence generated by the reaction of ATP and luciferin. Cells were plated at $5 \times 10^{4}$ cells per well on white 96-well microplates. Polyphenolics, pyruvate and toxic compound were added 24 before measurements. The final media volume was $200 \mu$ l. Luminescence levels were measured using a Tecan microplate reader (Tecan, Austria). All experiments were done in triplicate. Luciferase activity of untreated cells was arbitrarily set to $100 \%$. Data were expressed as \% of control ATP. 


\section{Results}

Firstly, to examine whether tested polyphenols and pyruvate possess potential in protection against chosen neurotoxins, we established dose-and time-response curves for 6-OHDA, 3-HK and salsolinol in cultures of SHSY5Y neuroblastoma cells. 6-OHDA, 3-HK and salsolinol induced cell death in a concentration-and a timedependent manner, as measured by MTT assay and CellTiter-Glo cell viability assay. An effective toxic dose of 6-OHDA after 24 hours treatment was observed at about $75 \mu \mathrm{M}$ in MTT assay and $50 \mu \mathrm{M}$ in ATP and CellTiter-Glo cell viability assay resulted in about $50 \%$ cell death. At $500 \mu \mathrm{M}$ concentration in MTT assay and 300 $\mu \mathrm{M}$ in CellTiter-Glo cell viability assay, 3-HK decreased cell viability to $\sim 50 \%$ of controls after exposure for 24 hours. Treatment of SH-SY5Y with salsolinol (500 $\mu \mathrm{M}$ or $250 \mu \mathrm{M}$, in MTT and CellTiter-Glo cell viability assay, respectively) for 24 hours significantly decreases cell viability to $50 \%$. Afterwards, SH-SY5Y cells were treated for 24 hours with wide range concentrations of each polyphenol and pyruvate. To determine whether tested substances need time to be able to penetrate into the cells, we also performed an additional experiment in which we added the phenolic compound or pyruvate to the culture cells for 24 hours before the toxic compound treatment.

The results of MTT cell viability assay demonstrated that luteolin at concentrations from $6 \mu \mathrm{M}$ to $13 \mu \mathrm{M}$ was the only phenolic compound that mitigated 6-OHDA and 3 -HK induced cell death when added simultaneously. Luteolin at $6 \mu \mathrm{M}$ restored the cell availability by 15 and at $13 \mu \mathrm{M}$ about $30 \%$ in both 6-OHDA and 3-HK toxicity model. Protective effects of luteolin against 3-HK and 6-OHDA are presented in Figure 1. Cytotoxic effect induced by salsolinol, wasn't inhibited by luteolin at none of the tested concentrations. Also preincubation with luteolin did not prevent 6-OHDA, 3-HK and salsolinol toxicity in the cells at any concentration. Furthermore, luteolin was found to be cytotoxic at concentrations above $25 \mu \mathrm{M}$ after 24 hours of treatment. After incubation with luteolin $25 \mu \mathrm{M}$ about $15 \%$ of dead cells were observed. It may suggest toxic effect of luteolin to the cells. Neither pre-treatment with various concentrations of other phenolic compounds nor simultaneous addition to SH-SY5Y cells had any protective effects in the 6-OHDA, salsolinol and 3-HK model of neurotoxicity. Additionally, sodium pyruvate was able to reverse the cytotoxicity caused by 6 -OHDA, 3-HK and salsolinol in a dose-dependent manner when pre-treated and cotreated. Treatment with pyruvate alone did not cause any viability loss up to the highest concentration $(1000 \mu \mathrm{M})$. As shown in Figure 2, treatment of SH-SY5Y cells with 100,500 and $1000 \mu \mathrm{M}$ of pyruvate reduced the 6-OHDA

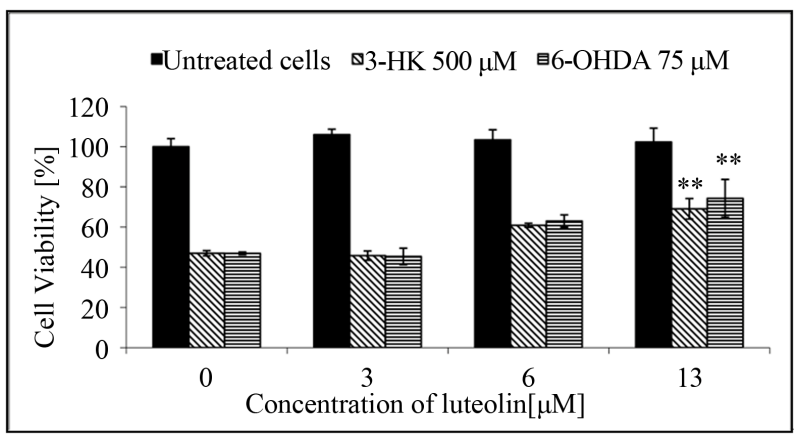

Figure 1. Luteolin protected SH-SY5Y neuroblastoma cells from 3-hydroxykynurenine (3-HK) and 6-hydroxydopamine (6-OHDA). SH-SY5Y human neuroblastoma cultures were exposed to 3-HK or 6-OHDA with or without various concentrations of luteolin for $24 \mathrm{~h}$ (3-HK, 6-OHDA), and cell viability was measured by the MTT assay and presented as untreated control. Significant neuroprotection was observed at 6 and $13 \mu \mathrm{M}$ of luteolin. Data points represent the mean \pm SEM of three independent experiments performed in triplicates. $* *: p<0.01$ compared to control cell death.

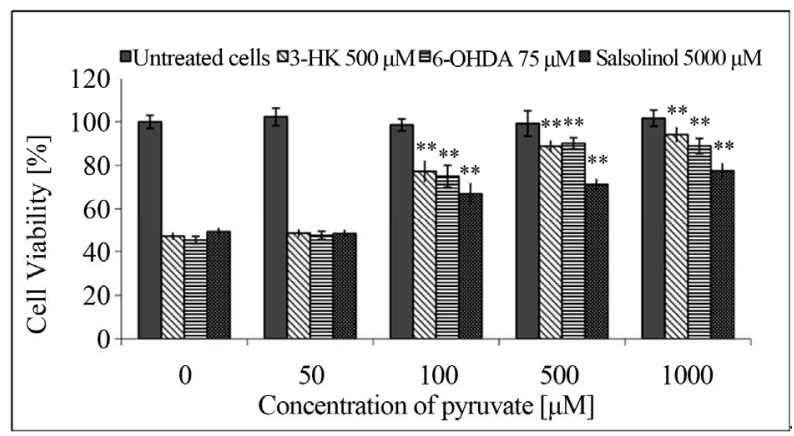

Figure 2. Pyruvate protected SH-SY5Y neuroblastoma cells from 3-hydroxykynurenine (3-HK), 6-hydroxydopamine (6-OHDA) and salsolinol toxicity. SH-SY5Y human neuroblastoma cultures were exposed to 3-HK, 6-OHDA and salsolinol with or without various concentrations of pyruvate for $24 \mathrm{~h}$ (3-HK, 6-OHDA, salsolinol) and cell viability was measured by the MTT assay and presented as untreated control. Significant neuroprotection was observed already at $100 \mu \mathrm{M}$ of pyruvate. Data points represent the mean \pm SEM of three independent experiments performed in triplicates. $* *: p<0.01$ compared to control cell death.

and 3 -HK cytotoxicity to about $77 \%, 75 \%, 89 \%, 90 \%$, $94 \%$ and $89 \%$ of the control group, respectively. Administration of pyruvate provided significant protection also against the toxicity of salsolinol, however 100, 500 and $1000 \mu \mathrm{M}$ pyruvate restored the MTT reduction from $49 \%$ to $67 \%, 71 \%$ and $78 \%$ of baseline, respectively. Interestingly, simultaneous addition of luteolin and pyruvate in the presence of toxic compound potentiated the protective effect. 3-HK $(500 \mu \mathrm{M})$ alone decreased cell viability to $47 \%$, with luteolin $(3 \mu \mathrm{M})$ and pyruvate (100 $\mu \mathrm{M})$ cell viability increased to $89 \%$, whereas pyruvate 
$(100 \mu \mathrm{M})$ alone increased to cell viability to $77 \%$ and luteolin $(3 \mu \mathrm{M})$ wasn't found to be able to restore cell viability at all. At higher concentrations of pyruvate (500 $\mu \mathrm{M})$ and luteolin $(6 \mu \mathrm{M})$ cell viability was almost completely restored. This finding we report for the first time. Effect of pyruvate on 3-HK toxicity on neuroblastoma cells is presented in Figure 3.

The effects of 6-OHDA, 3-HK and salsolinol on intracellular ATP content in SH-SY5Y cells were assessed using CellTiter-Glo cell viability assay after 24 hours. As expected, 6-OHDA, 3-HK and salsolinol induced a dosedependent decrease in cellular ATP content. The decrease in intracellular level of cellular ATP induced by 6-OHDA and 3-HK was attenuated after $24 \mathrm{~h}$ pre-treatment with similar concentrations of luteolin $(6-13 \mu \mathrm{M})$ and pyruvate $(100 \mu \mathrm{M}, 500 \mu \mathrm{M}, 1000 \mu \mathrm{M})$ to those found in MTT-assay, as shown in Figures 4 and 5, respectively. However, salsolinol induced toxicity was also reduced in the presence of pyruvate but not by luteolin. Interestingly, after SH-SY5Y cells were treated for 24 hours with various concentrations of luteolin alone, increase in the mitochondrial ATP level was observed as compared to untreated cells. At concentration of $3 \mu \mathrm{M}$ and $6 \mu \mathrm{M}$ luteolin ATP-levels were $113 \%$ and $117 \%$. However, ATP-level after luteolin treatment at concentration $13 \mu \mathrm{M}$ returned to $106 \%$.

Co-treatment of $6 \mu \mathrm{M}$ luteolin and $100 \mu \mathrm{M}$ pyruvate increased cellular ATP concentration in the presence of 6-OHDA and 3-HK (Figure 6). Moreover, $6 \mu \mathrm{M}$ luteolin and $500 \mu \mathrm{M}$ pyruvate elevated ATP level in SH-SY5Y to

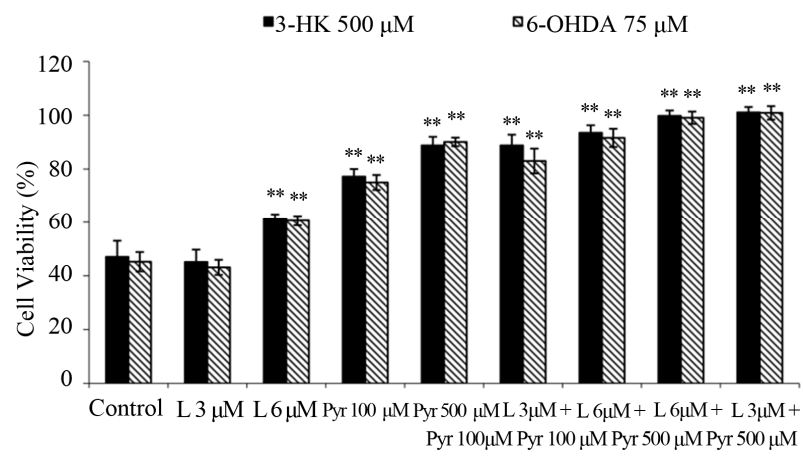

Figure 3. Coadministration of luteolin and pyruvate enhanced protection of SH-SY5Y neuroblastoma cells from 3-hydroxykynurenine (3-HK) and 6-hydroxydopamine (6OHDA) toxicity. SH-SY5Y human neuroblastoma cultures were exposed to 3-HK and 6-OHDA with or without various concentrations of luteolin (L) and pyruvate (Pyr) for $24 \mathrm{~h}$ (3-HK, 6-OHDA), and cell viability was measured by the MTT assay and presented as untreated controls. Significant neuroprotection was observed already at $100 \mu \mathrm{M}$ of pyruvate and $3 \mu \mathrm{M}$ luteolin. Data points represent the mean \pm SEM of three independent experiments performed in triplicates. ${ }^{* *}: \mathrm{p}<0.01$ compared to control cell death.

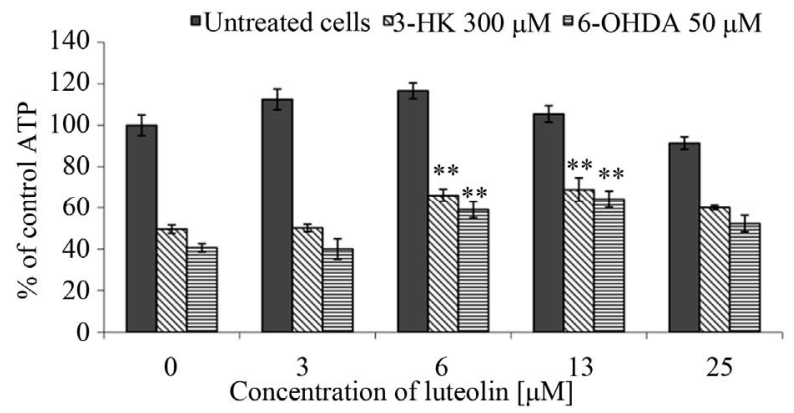

Figure 4. Luteolin increased mitochondrial ATP level in 3-hydroxykynurenine (3-HK) and 6-hydroxydopamine (6OHDA) in SH-SY5Y neuroblastoma cells induced neurotoxicity. SH-SY5Y human neuroblastoma cultures were exposed to 3-HK or 6-OHDA with or without various concentrations of luteolin for $24 \mathrm{~h}$ (3-HK, 6-OHDA), and ATPlevel was measured by the CellTiter-Glo cell viability assay and presented as untreated control. Significant ATP-level increase was observed at 6 and $13 \mu \mathrm{M}$ of luteolin. Data points represent the mean \pm SEM of three independent experiments performed in triplicates. $* *: p<0.01$ compared to control cell death.

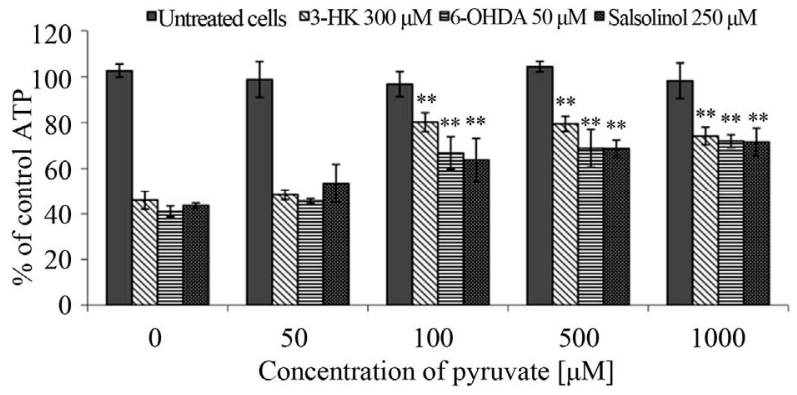

Figure 5. Pyruvate increased mitochondrial ATP level in 3-hydroxykynurenine (3-HK) and 6-hydroxydopamine (6OHDA) in SH-SY5Y neuroblastoma cells induced neurotoxicity. SH-SY5Y human neuroblastoma cultures were exposed to 3-HK, 6-OHDA and salsolinol with or without various concentrations of pyruvate for $24 \mathrm{~h}$ (3-HK, 6OHDA, salsolinol), and ATP-level was measured by the CellTiter-Glo cell viability assay and presented as untreated control. Significant ATP-level was observed already at 100 $\mu M$ of pyruvate. Data points represent the mean \pm SEM of three independent experiments performed in triplicates. **: $p<0.01$ compared to control cell death.

near $100 \%$ and completely blocked the 3 -HK and $6-$ OHDA toxicity.

\section{Discussion}

In the present study we intended to evaluate structurally diverse plant phenolics for their abilities to reduce neurotoxicity induced by substances with different mode of action in SH-SY5Y human neuroblastoma cell line. The results of MTT and CellTiter-Glo cell viability assay show that most of the tested polyphenolic compounds 


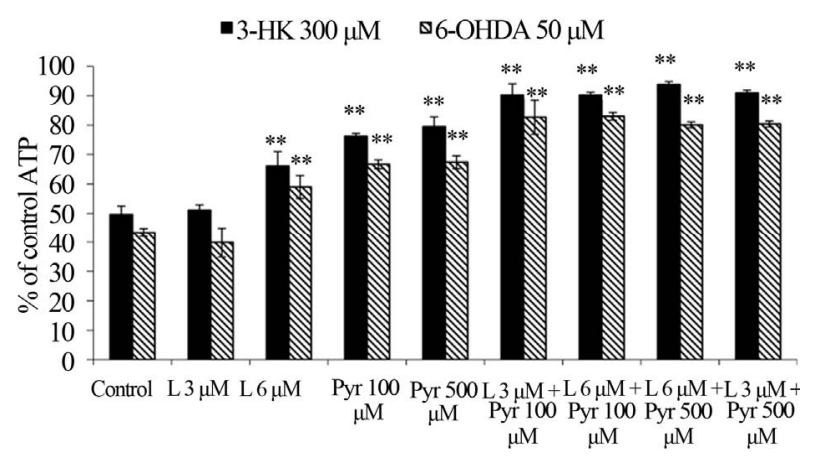

Figure 6. Coadministration of luteolin and pyruvate enhanced mitochondrial ATP-level in SH-SY5Y neuroblastoma cells induced 3-hydroxykynurenine (3-HK) and 6-hydroxydopamine (6-OHDA) toxicity. SH-SY5Y human neuroblastoma cultures were exposed to 3-HK and 6-OHDA with or without various concentrations of luteolin (L) and pyruvate (Pyr) for $24 \mathrm{~h}$ (3-HK, 6-OHDA), and ATP-level was measured by the CellTiter-Glo cell viability assay and presented as untreated controls. Significant neuroprotection was observed already at $100 \mu \mathrm{M}$ of pyruvate and $3 \mu \mathrm{M}$ luteolin. Data points represent the mean \pm SEM of three independent experiments performed in triplicates. $* *: p<$ 0.01 compared to control cell death.

failed to protect against 6-OHDA, 3-HK and salsolinol induced toxicity. Antioxidative efficacy of flavonoids and polyphenolic acids in vitro are well described in the literature [15-17]. Ishige et al., 2001 suggested that in the protective properties of flavonoids three different mechanisms are implicated, namely increasing intracellular glutathione, lowering levels of ROS and preventing the influx of $\mathrm{Ca}^{2+}$ [18]. However, in order to display neuroprotective activity a potent protectant need to enter a neuronal cell through the cell membrane. Our results would indicate that most of the tested polyphenolics enter the cell rather poorly, if at all and due to this fact is not able to achieve a protective level of concentration. Among the tested phenolic compounds, only luteolin exhibited neuroprotection against oxidative stress-induced by 6-OHDA and 3-HK by increasing cell survival and intracellular ATP level in SH-SY5Y human neuroblastoma cell line. However, no cell survival and increase in ATP level was observed in the presence of salsolinol. In this investigation several neurotoxins were chosen as oxidative stressors. Salsolinol has been reported to cause oxidative stress by increasing ROS levels, decreasing ATP and glutathione levels in SH-SY5Y cell line by inhibiting mitochondrial complex-I and complex-II enzyme activities, tyrosine hydroxylase and monoamine oxidase $[7,19,20]$. Cell death was not inhibited by the addition of antioxidants such as $\alpha$-tocopherol or the water-soluble vitamin $\mathrm{E}$ analogue-Trolox $\mathrm{C}$, however nicotine and donepezil restored cell survival, indicating that in the salsolinol toxicity nicotinic receptors could be involved
$[21,22]$. Another toxic insult used in this investigation is the widely used dopamine derivative 6-OHDA, which similarly to salsolinol induces ROS generation and inhibits mitochondrial complex I and, additionally, mitochondrial complex IV [23]. On the other hand, 3-HK is a kynurenine pathway intermediate, which neurotoxicity results from the intracellular accumulation of hydrogen peroxide [24]. Based on available elusive evidences, it's difficult to explain the lack of neuroprotective activity of luteolin against salsolinol induced cell death. Probably in the cell death induced by salsolinol different signaling pathways are involved and luteolin is not able to interact with cytotoxic mechanisms caused by this neurotoxin.

In our study, when the cells were preincubated with luteolin and then exposed to 3-HK or 6-OHDA, we could not observe protection against these toxic compounds. Probably, the hydroxyl groups present in the luteolin molecule were metabolized or oxidized. Luteolin (3', 4', 5, 7-tetrahydroxyflavone) is a widespread flavonoid aglycone found in high concentrations in celery (Apium graveolens), peppers (Capsicum annuum), perilla (Perilla frutescens) and has been shown to posses many biological activities including antioxidant $[25,26]$ and antiinflammatory $[27,28]$. Luteolin bears an electrophilic $\alpha-\beta$-unsaturated carbonyl moiety with intermediate polarity and solubility in DMSO. Furthermore, also tested sinensetin - a flavone of similar structure to luteolin but fully methylated was not found to be neuroprotective in none of the experiments. On the other hand, apigenin and chrysin belonged to the same large group of flavones and were not found to be protective. Moreover, also tested cynaroside, a 7-O-glucoside of luteolin has not been found to reduce induced toxicity. These findings suggest that presence of free hydroxyl group on $\mathrm{C} 7$ and in the position $\mathrm{C}^{\prime}$ ' and $\mathrm{C}^{\prime}$ ' on the B-ring play an important role in acting as neuroprotectant and would support our hypothesis that the ability of luteolin to exhibit neuroprotection in the 6-OHDA, 3-HK and induced cell death may be related to the capability to penetrate into the cell. This result was in agreement with previous studies, where the authors suggested that presence of catecholo-dihydroxy structure on the B-ring build by C' 3 and C' 4 is crucial for scavenger properties [15].

On the other hand, numerous findings indicated that neither lowering ROS nor increasing antioxidant capacity were not able to bring health-beneficial effects [29,30]. On the contrary, it has been postulated that ROS formation might induce cellular signalling, transmit messages between mitochondria and other compartments and increase activities of phase II response enzymes that protect from ROS damage $[31,32]$. This theory could partly explain the lack of cell death protecting activities of polyphenols presented in this investigation. However, it also has to be mentioned that perhaps the benefits of 
ROS formation might not be true for neurons, which contain more mitochondria than other cells.

Our study has also revealed that pyruvate could act as a survival factor protecting SH-SY5Y cells from 6OHDA and 3-HK and salsolinol injury. Enhancing of neuronal defense of pyruvate against 6-OHDA induced cell death has been reported before [12], however, reduced neurotoxic action by pyruvate against $3-\mathrm{HK}$ and salsolinol induced apoptosis we report for the first time. Interestingly, increasing concentrations of pyruvate showed no further improvement of protective effects against 3-HK, 6-OHDA and salsolinol. Furthermore, in our study administration of both pyruvate and luteolin resulted unexpectedly in additive neuroprotective effect against 6-OHDA and 3-HK-toxicity. This finding suggests that luteolin and pyruvate posses different cell survival-increasing activities. Further investigations are required to determine what is responsible for the observed additive effect of luteolin and pyruvate. Briefly, pyruvate is a final product of glycolysis, which enters the Krebs cycle and is a source of ATP generation. Moreover, pyruvate has been reported to upregulate glutathione peroxidase expression and to activate the Akt signaling pathway [10].

We couldn't confirm actions of EGCG against 3-HK induced neurocytotoxicity in SH-SY5Y cell line reported earlier by Jeong et al. [33]. At the same reported concentration of $10 \mu \mathrm{M}$ no cytoprotective effects were observed [33]. Neuroprotection had been also described for estrogens [34]. However, kaempferol-a flavonol with estrogenic and antiestrogenic properties was not found to be protective against neurotoxicity in our investigation as it was in the study conducted by Roth et al., on PC12 rat pheochromocytoma cell line [35]. Also rosmarinic acid was not observed to reduce cell death in 6-OHDA treated MES23.5 dopaminergic cells. These may be due to the fact that in those studies different cell lines as models of neurotoxicity were used.

In conclusion, we found that the combined administration of luteolin and pyruvate exerted neuroprotective effects against 6-OHDA and 3-HK-toxicity by increasing cellular ATP levels and number of surviving cells. However, only pyruvate was found to enhance neuronal defence against salsolinol-induced cell-death. Therefore, luteolin in combination with pyruvate can become interesting candidate for possible treatment of neuronal injury. Further studies are necessary to determine what is responsible for the observed additive protective effect of luteolin and pyruvate.

\section{REFERENCES}

[1] M. T. Lin and M. F. Beal, "Mitochondrial Dysfunction and Oxidative Stress in Neurodegenerative Diseases,"
Nature, Vol. 443, No. 7113, 2006, pp. 787-795.

doi:10.1038/nature05292

[2] L. M. Sayre, G. Perry and M. A. Smith, "Oxidative Stress and Neurotoxicity," Chemical Research in Toxicology, Vol. 21, No. 1, 2008, pp. 172-188.

doi:10.1021/tx700210j

[3] C. Behl, J. B. Davis, R. Lesley and D. Schubert, "Hydrogen Peroxide Mediates Amyloid $\beta$-Protein Toxicity," Cell, Vol. 77, No. 6, 1994, pp. 817-827. doi:10.1016/0092-8674(94)90131-7

[4] M. F. Galindo, J. Jordan, C. Gonzales-Garcia and V. Cena, "Chromaffin Cell Death Induced by 6-Hydroxydopamine Is Independent of Mitochondrial Swelling and Caspase Activation," Journal of Neurochemistry, Vol. 84, No. 5, 2003, pp. 1066-1073. doi:10.1046/j.1471-4159.2003.01592.x

[5] N. Li, K. Ragheb, G. Lawler, J. Sturgis, B. Rajwa, J. A. Melendez and J. P. Robinson, "Mitochondrial Complex I Inhibitor Rotenone Induces Apoptosis through Enhancing Mitochondrial Reactive Oxygen Species Production," The Journal of Biological Chemistry, Vol. 278, No. 10, 2003, pp. 8516-8525. doi:10.1074/jbc.M210432200

[6] K. Shimoke, M. Kudo and T. Ikeuchi, "MPTP-Induced Reactive Oxygen Species Promote Cell Death through a Gradual Activation of Caspase-3 without Expression of GRP78/BiP as a Preventive Measure against ER Stress in PC12 Cells," Life Sciences, Vol. 73, No. 5, 2003, pp. 581593. doi:10.1016/S0024-3205(03)00351-5

[7] S. Wanpen, P. Govitrapong, S. Shavali, P. Sangchot and M. Ebad, "Salsolinol, a Dopamine-Derived Tetrahydroisoquinoline, Induces Cell Death by Causing Oxidative Stress in Dopaminergic SH-SY5Y Cells and the Said Effect Is Attenuated by Metallothionein," Brain Research, Vol. 1005, No. 1-2, 2004, pp. 67-76. doi:10.1016/j.brainres.2004.01.054

[8] C. R. Evans, "Flavonoid Antioxidants," Current Medicinal Chemistry, Vol. 8, No. 7, 2001, pp. 797-807. doi:10.2174/0929867013373011

[9] Y. Xu, S. Sun, X. Cao and E. Tong, "Protective Effect of GSH on PD Model Induced by 6-OHDA in Vitro," Journal of Huazhong University of Science and Technology, Vol. 22, No. 4, 2002, pp. 355-358. doi:10.1007/BF02896785

[10] F. J. Fernandez-Gomez, M. D. Pastor, E. M. Garcia-Martinez, R. Melero-Fernandez de Mera, M. Gou-Fabregas, M. Gomez-Lazaro, S. Calvo, R. M. Soler, M. F. Galindo and J. Jordan, "Pyruvate Protects Cerebellar Granular Cells from 6-Hydroxydopamine Induced Cytotoxicity by Activating the Akt Signalling Pathway and Increasing Glutathione Peroxidise Expression," Neurobiology of Disease, Vol. 24, No. 2, 2006, pp. 296-307. doi:10.1016/j.nbd.2006.07.005

[11] A. M. Munoz, P. Rey, R. Soto-Otero, M. J. Guerra and J. L. Labandeira-Garcia, "Systemic Administration of N-Acetylcysteine Protects Dopaminergic Neurons against 6-Hydroxydopamine-Induced Generation," Journal of Neuroscience Research, Vol. 76, No. 4, 2004, pp. 551-562. doi:10.1002/jnr.20107 
[12] X. Wang, E. Perez, R. Liu, R. T. Mallet and S. H. Yang, "Pyruvate Protects Mitochondria from Oxidative Stress in Human Neuroblastoma SK-N-SH Cells," Brain Research, Vol. 1132, No. 1, 2007, pp. 1-9. doi:10.1016/j.brainres.2006.11.032

[13] G. Alvarez, M. Ramos, F. Ruiz, J. Satrustegui and E. Bogonez, "Pyruvate Protection against $\beta$-Amyloid-Induced Neuronal Death: Role of Mitochondrial Redox State," Journal of Neuroscience, Vol. 73, No. 2, 2003, pp. 260269. doi:10.1002/jnr. 10648

[14] T. Mosmann, "Rapid Colorimetric Assay for Cellular Growth and Survival: Application to Proliferation and Cytotoxicity Assays," Journal of Immunological Methods, Vol. 65, No. 1-2, 1983, pp. 55-63. doi:10.1016/0022-1759(83)90303-4

[15] C. A. Rice Evans, N. J. Miller and G. Paganga, "Structure-Antioxidant Activity Relationships of Flavonoids and Phenolic Acids," Free Radical Biology and Medicine, Vol. 36, No. 7, 1996, pp. 933-956. doi:10.1016/0891-5849(95)02227-9

[16] P. Otero, M. Viana, E. Herrera and B. Bonet, "Antioxidant and Prooxidant Effects of Ascorbic Acid, Dehydroascorbic Acid and Flavonoids on LDL Submitted to Different Degrees of Oxidation," Free Radical Biology and Medicine, Vol. 27, No. 6, 1997, pp. 619-626. doi:10.3109/10715769709097865

[17] P. G. Pietta, "Flavonoids as Antioxidants," Journal of Natural Product, Vol. 63, No. 7, 2000, pp. 1035-1042. doi:10.1021/np9904509

[18] K. Ishige, D. Schubert and Y. Sagara, "Flavonoids Protect Neuronal Cells from Oxidative Stress by Three Distinct Mechanisms," Free Radical Biology and Medicine, Vol. 30, No. 4, 2001, pp. 433-446. doi:10.1016/S0891-5849(00)00498-6

[19] M. E. Bembenek, C. W. Abell, L. A. Chrisey, M. D. Rozwadowska, A. W. Gessner and A. Brossi, "Inhibition of Monoamine Oxidase-A and Oxidase-B by Simple Isoquinoline Alkaloids-Racemic and Optically Active 1,2,3,4Tetrahydroisoquinoline, 3,4-Dihydroisoquinoline, and Fully Aromatic Isoquinoline," Journal of Medical Chemistry, Vol. 33, No. 1, 1990, pp. 147-152. doi:10.1021/jm00163a025

[20] A. Storch, A. Kaftan, K. Burkhardt and J. Schwarz, "1-Methyl-6,7-dihydroxy-1,2,3,4-tetrahydroisoquinoline Salsolinol Is Toxic to Dopaminergic Neuroblastoma SH-SY5Y Cells via Impairment of Cellular Energy Metabolism," Brain Research, Vol. 855, No. 1, 2000, pp. 67-75. doi:10.1016/S0006-8993(99)02272-6

[21] J. M. Willets, D. G. Lambert, J. Lunec and H. R. Griffiths, "Studies on the Neurotoxicity of 6,7-Dihydroxy-1-methyl1,2,3,4-tetrahydroisoquinoline (Salsolinol) in SH-SY5Y Cells," European Journal of Pharmacology: Environmental Toxicology and Pharmacology, Vol. 293, No. 4, 1995, pp. 319-326. doi:10.1016/0926-6917(95)90051-9

[22] D. J. Bonda, M. Mailankot, J. G. Stone, M. R. Garrett, M. Staniszewska, R. J. Castellani, J. R. Das and Y. Tizabi, "Additive Protective Effects of Donezepil and Nicotine against Salsolinol-Induced Cytotoxicity in SH-SY5Y Cells," Neurotoxicity Research, Vol. 16, No. 3, 2009, pp.
194-204.

[23] Y. Y. Glinka and M. B. Youdim, "Inhibition of MitoChondrial Complexes I and IV by 6-Hydroxydopamine," European Journal of Pharmacology: Environmental Toxicology and Pharmacology, Vol. 292, No. 3-4, 1995, pp. 329-332.

[24] C. L. Eastman and T. R. Guilarte, "The Role of Hydrogen Peroxide in the in Vitro Cytotoxicity of 3-Hydroxykynurenine," Neurochemical Research, Vol. 15, No. 11, 1990, pp. 1101-1107. doi:10.1007/BF01101711

[25] V. Manju, V. Balsubramaniyan and N. Nalini, "Rat Colonic Lipid Peroxydation and Antioxidant Status: The Effects of Dietary Luteolin in 1,2-Dimethylhydrazine Challenge," Cellular \& Mollecular Biology Letters, Vol. 10, No. 3, 2005, pp. 535-551.

[26] Z. Quisheng, Z. Yuntao, Z. Rongliang, G. Dean and L. Changling, "Effects of Verbascoside and Luteolin on Oxidative Damage in Brain of Heroin Treated Mice," Pharmazie, Vol. 60, No. 7, 2005, pp. 539-543.

[27] J. S. Kim and C. Jobin, "The Flavonoid Luteolin Prevents Lipopolysaccharide-Induced NF-KappaB Signalling and Gene Expression by Blocking IkappaB Kinase Activity in Intestinal Epithelial Cells and Bone-Marrow Derived Dendritic Cells," Immunology, Vol. 115, No. 3, 2005, pp. 375-387. doi:10.1111/j.1365-2567.2005.02156.x

[28] C. Y. Chen, W. H. Peng, K. D. Tsai and S. L. Hsu, "Luteolin Suppresses Inflammation-Associated Gene Expression by Blocking NF-KappaB and AP-1 Activation Pathway in Mouse Alveolar Macrophages," Life Sciences, Vol. 81, No. 23-24, 2007, pp. 1602-1614. doi:10.1016/j.lfs.2007.09.028

[29] N. R. Cook, C. M. Albert, J. M. Gaziano, E. Zaharris, J. MacFadyen, E. Danielson, J. Buring and J. E. Manson, "A Randomized Factorial Trial of Vitamins X and E and Beta Carotene in the Secondary Prevention of Cardiovascular Events in Women: Results from the Women's Antioxidant Cardiovascular Study," Archives of Internal Medicine, Vol. 167, No. 15, 2007, pp. 1610-1618. doi:10.1001/archinte.167.15.1610

[30] N. Katsiki and C. Manes, "Is There a Role for Supplemented Antioxidants in the Prevention of Artherioscleriosis?" Clinical Nutrition, Vol. 28, No. 1, 2009, pp. 3-9. doi:10.1016/j.clnu.2008.10.011

[31] R. D. Guzy and P. T. Schumacker, "Oxygen Sensing by Mitochondria at Complex III: The Paradox of Increased Reactive Oxygen Species during Hypoxia," Experimental Physiology, Vol. 91, No. 5, 2006, pp. 807-819. doi:10.1113/expphysiol.2006.033506

[32] E. Owusu-Ansah, A. Yavari, S. Mandal and U. Banerjee, "Distinct Mitochondrial Retrograde Signals Control the G1-S Cell Cycle Checkpoint," Nature Genetics, Vol. 40, No. 3, 2008, pp. 356-361. doi:10.1038/ng.2007.50

[33] J. H. Jeong, H. J. Kim, T. J. Lee, M. K. Kim, E. S. Park and B. S. Choi, "Epigallocatechin-3-Gallate Attenuates Neuronal Damage Induced by 3-Hydroxykynurenine," Toxicology, Vol. 195, No. 1, 2004, pp. 53-60. doi:10.1016/j.tox.2003.08.007

[34] C. Behl, T. Skutella, F. Lezoualch, A. Post, M. Widmann, 
C. J. Newton and F. Holsboer, "Neuroprotection against Oxidative Stress by Estrogens: Structure-Activity Relationship," Molecular Pharmacology, Vol. 51, No. 4, 1997, pp. 535-541.

[35] A. Roth, W. Schaffner and C. Hertel, "Phytoestrogen Kaempferol (3,4',5,7-Tetrahydroxyflavone) Protects PC12 and T47D Cells from $\beta$-Amyloid-Induced Toxicity," Journal of Neuroscience Research, Vol. 57, No. 3, 1999, pp. 399-404.

doi:10.1002/(SICI)1097-4547(19990801)57:3<399::AIDJNR12>3.0.CO;2-W 